\title{
Espiritualidad en la Tercera Edad
}

\author{
Cecilia San Martín Petersen *
}

\section{Resumen}

La espiritualidad puede entenderse como un conjunto de sentimientos, creencias y acciones que suponen una búsqueda de lo trascendente, sagrado o divino. En tanto representaciones acerca de un poder final último, contribuyen a dar un sentido y propósito en la vida, y orientan la conducta de las personas, sus relaciones interpersonales y su forma de sentir y de pensar, tanto a la realidad como a sí mismos.

Dado que tanto en el proceso de envejecer como en la evaluación de la vida que suele ocurrir al aproximarse a la muerte, pueden surgir conflictos, confusión y sufrimiento, la concepción que las personas tengan acerca de lo que hay más allá de la muerte, o las respuestas que se hayan dado a las preguntas de por qué y para qué de la vida, resultan determinantes en el bienestar psíquico de los adultos mayores.

Si además se considera que la expectativa de vida ha aumentado considerablemente, y está cambiando la manera de envejecer, en qué consiste ser viejo, y las ventajas y desventajas o problemas de este proceso en sus distintos contextos, es necesario repensar la vejez, tanto como etapa de vida, como en las políticas que afectan la calidad y condiciones de vida de este sector de la población.

Por lo tanto, se puede sostener que todo profesional que trabaja con adultos mayores, y en particular los profesionales de la salud mental, deben poder considerar los referentes espirituales del propio individuo para ayudarlo de buena forma con los problemas que puedan surgir en el proceso de envejecimiento.

Palabras clave: espiritualidad, tercera edad, bienestar psíquico.

\section{Abstract}

Spirituality may be understood as a group of feelings, beliefs and actions that suppose a search for the transcendent, sacred or divine. As representations about an ultimate power, they contribute to the sense and purpose in life and orient peoples behavior, relationships, and ways to feel and think about reality and about themselves.

Since either in the growing old process and in the evaluation of life that occurs when approaching to death it may emerge conflicts, confusion and suffering, people beliefs about what is beyond death, or the answers to the questions about what for and why of life, become determinants in elders well-being.

* Universidad de La Frontera, Temuco, Chile. Doctoranda. Doctorado en Psicología UP. e-mail: csmartin@ufro.cl 
Furthermore, considering that life expectancy has significantly increased, and that the ways of growing old are changing as well as what being old means, and this process advantages and disadvantages or problems, in it's different contexts, it's necessary to think old age over again, as well as the policies that affect the quality of life of this group of people.

Therefore, every professional who assist elderly, specially mental health professionals, must consider the spiritual referents of the individual in order to give the best assistance in whatever problems may appear in the growing old process.

Key words: Spirituality, elderly, psychological well-being. 


\section{Introducción}

Durante las últimas décadas la esperanza de vida ha crecido de tal modo que se observa un envejecimiento de la población nunca antes visto. Este hecho ha resaltado la necesidad de pensar de nuevo la vejez y el envejecimiento, pues no se tiene la certeza de que los conocimientos previos, surgidos cuando la vejez abarcaba una pequeña porción de la vida que sólo alcanzaban unos pocos, resulten adecuados en la actualidad, en que un número considerable de personas llega a esta etapa, la cual puede abarcar un tercio de sus vidas.

El bienestar personal, conformado por los denominados bienestar subjetivo y bienestar psicológico (Tonon, 2006), es uno de los aspectos de la calidad de vida de los adultos mayores que requiere ser revisado. Este trabajo se focaliza en la espiritualidad en la tercera edad, por su relación con varias de las dimensiones del bienestar psicológico, tales como autoaceptación, relación con los demás, desarrollo y crecimiento personal, y propósito en la vida.

La espiritualidad es uno de los campos que está siendo estudiado muy recientemente, de manera científica, fundamentalmente en el campo de la salud, y específicamente de la salud mental. Es interesante notar que entre los intereses iniciales de la psicología se encontraba la espiritualidad (Pargament \& Mahoney, 2002; Spilka, 2003), y que a partir del siglo XX tiende a ser ignorada por los psicólogos, que la conciben como algo patológico o como un proceso que puede reducirse a funciones psicológicas, sociales y biológicas subyacentes más básicas (Pargament \& Mahoney, 2002).

En gran medida gracias al impulso dado por la psicología positiva, a fines del siglo $\mathrm{XX}$ se retoman las tres misiones originales de la psicología: la cura de los trastornos mentales, el hacer la vida de las personas más productivas y plenas, e identificar y fortalecer a las personas talentosas. De este modo, abundante investigación se está llevando a cabo acerca de temas como las emociones positivas, los rasgos positivos del carácter, instituciones positivas, etc. (Peterson \& Seligman, 2004). Y pareciera que, finalmente, las variadas razones por las cuales la comunidad científica dejó de lado la espiritualidad como una importante dimensión humana han comenzado a superarse (Koenig, 2001; Kliewer \& Saultz, 2006; Levin, 2001).

\section{Espiritualidad}

Algunos autores no distinguen entre religión y espiritualidad, y otros los diferencian, para algunos son constructos radicalmente distintos mientras que para otros están relacionados. Para Rivera-Ledesma y Montero-López (2005), a pesar de que ambas son convicciones espirituales y estilos de vida, hay diferencias entre religiosidad y espiritualidad. La religiosidad es de naturaleza esencialmente social y se vive como un cuerpo de conocimientos, ritos, normas y valores que rigen la vida del individuo interesado en vincularse con lo divino, mientras que la naturaleza de la espiritualidad es singular, específica y personal, se caracteriza por un sentimiento de integración con 
la vida y con el mundo, y se vive como la experiencia de lo divino. La religiosidad tendría un carácter directivo, al señalar cómo buscar lo divino, a través del adoctrinamiento y la congregación con otros, mientras que la espiritualidad se caracterizaría por la búsqueda de lo sacro o divino a través de cualquier ruta o experiencia de la vida (Kliewer \& Staultz, 2006; Rivera-Ledesma \& Montero-López, 2007).

De modo similar, Green, Benshoff, y Harris-Forbes (2001), refieren que se espera que los adherentes a una religión comulguen o expresen su devoción colectivamente en la iglesia, sinagoga, o mezquita, siguiendo un ritual preestablecido, y orando conforme a dogmas estrictos. Por su parte, al ser la espiritualidad un acto más privado, tiende a evitar las estructuras externas formales de los rituales. No obstante, estos autores plantean que la espiritualidad es parte de la mayoría de las religiones, y cada religión monoteísta comprende elementos espirituales, tanto para la devoción colectiva, como para las prácticas religiosas individuales. Por tanto, se puede sostener que ser religioso es a menudo ser espiritual, a lo menos parcialmente, pero el ser espiritual no implica necesariamente ser religioso.

Se ha sugerido que la religión es institucional, dogmática, y restrictiva, mientras que la espiritualidad sería personal, subjetiva y fomentadora de la calidad de vida. Sin embargo, Pargament y Mahoney (2002) señalan la conveniencia de no polarizar los dos constructos, y destacan que hay importantes puntos de solapamiento entre ambos.

Tanto religiosidad como espiritualidad se pueden expresar social e individualmente, y ambas tienen la capacidad de promover o impedir el bienestar. Estos autores prefieren utilizar el término religión en su sentido más clásico, de un amplio dominio individual e institucional, que sirve distintos objetivos, tanto seculares como sagrados; y definir espiritualidad como la búsqueda de lo sagrado (Pargament \& Mahoney, 2002). Pargament (1997, citado en Peterson \& Seligman, 2004), señala que así como la espiritualidad es la búsqueda de lo sagrado, la religión es la búsqueda de significado en formas relacionadas con lo sagrado. De este modo, la espiritualidad es el corazón de la religión, y su más importante función.

Peterson y Seligman (2004), plantean que tanto espiritualidad como religiosidad hacen referencia a las creencias y prácticas basadas en la convicción de que existe una dimensión trascendente, no física, de la vida. Estas creencias son persuasivas, abarcadoras y estables, y brindan información acerca de las atribuciones que realizan las personas, los significados que construyen y las formas en que se interrelacionan con otros. La espiritualidad sería universal, todas las culturas tienen concepciones y representaciones referidas a una fuerza última, trascendente y sagrada, que orienta en los temas centrales de la vida, como el sentido y propósito de la vida, y ofrecen reglas y valores que guían las relaciones interpersonales.

En el mismo sentido, Benjamin y Looby (1998, citados en Green, Benshoff, \& Harris-Forbes, 2001), plantean que la religión puede no ser universal, pero la espiritualidad si lo es, y existe tanto fuera como dentro de contextos religiosos y adopta su forma específica de acuerdo con la cultura en que se manifiesta. Para Kliewer y Staultz (2006), cada persona tiene su particular cultura espiritual que es producto de la 
combinación de su espiritualidad personal y de las experiencias espirituales o esquemas culturales a los que ha sido expuesto.

Para Delgado (2005), la espiritualidad va más allá de la religión y de los límites culturales, y se caracterizaría por la fe, por un sentido de significado y propósito en la vida, un sentido de conexión con los demás y una trascendencia de sí mismo, las que resultan en bienestar y paz interior. En un sentido similar, el instituto nacional para el cáncer de Estados Unidos (National Cancer Institute (s/f), define la espiritualidad como profundos sentimientos y creencias, a menudo religiosas, que incluyen una sensación personal de paz, propósito, conexión con otros, y creencias acerca del significado de la vida.

Al entender espiritualidad como la búsqueda de lo sagrado, Pargament y Mahoney (2002) señalan que se tiene una definición lo suficientemente amplia como para abarcar tanto las expresiones espirituales tradicionales, teocéntricas y de base institucional, como las expresiones no-teístas, apartadas de las creencias y prácticas tradicionales. Y a la vez, permite diferenciar el constructo espiritualidad de otras manifestaciones relacionadas, al centrarla en torno a la percepción de lo sagrado. Estos autores explican que la espiritualidad puede ser usada tanto constructiva como destructivamente, por lo que no es inherentemente buena o mala, y su valor depende de la manera específica en que las personas buscan lo sagrado (Pargament \& Mahoney, 2002).

Espiritualidad como búsqueda de lo sagrado implica entonces que ésta es un proceso, e incluye tanto los esfuerzos por descubrir lo sagrado, como los esfuerzos por conservarlo una vez encontrado. De este modo, una persona podría ser descrita como espiritual en la medida que trata de encontrar, saber, experienciar, o relacionarse con aquello que percibe como sagrado. Además de tratarse de un proceso, la espiritualidad puede entenderse y ser evaluada como un resultado, obtenido naturalmente a través de la búsqueda de lo sagrado, como la paz o el bienestar espiritual (Pargament \& Mahoney, 2002).

Aunque desde las ciencias sociales no es posible hablar de la existencia real de lo sagrado o de Dios, si resulta posible y deseable conocer acerca de la variedad de formas en que las personas tratan de descubrir y conservar lo que perciben como sagrado (Pargament \& Mahoney, 2007). No se trata de establecer la validez de la cura por la fe, sino de investigar el poder terapéutico o sanador de la fe de las personas (Koenig, 2001).

Existen ilimitadas maneras para descubrir y conservar lo sagrado. Estas van desde involucrarse en instituciones religiosas tradicionales, a grupos, programas y asociaciones espirituales no tradicionales. También sistemas de creencias que van desde las religiones tradicionales organizadas, modernos movimientos espirituales, a visiones de mundo más individuales. Y en cuanto a prácticas, están desde las religiosas más tradicionales de oración, lectura de la Biblia, ritos de pasaje, hasta otras expresiones humanas que tienen también como meta lo sagrado, tales como yoga, música, arte, etc. (Pargament \& Mahoney, 2002).

Se conocen hoy buenas razones por las cuales la espiritualidad debe ser estudiada. Por una parte, las creencias y experiencias religiosas y espirituales son parte integrante de la personalidad (Peterson \& Seligman, 2004). Por otra parte, un cuerpo creciente de 
investigación empírica está mostrando importantes alcances de la espiritualidad en variados aspectos de las personas. Pargament y Mahoney (2002), citan estudios que demuestran implicancias positivas de la espiritualidad en salud mental, uso de alcohol y drogas, ajuste marital, crianza, consecuencias de experiencias de vida estresantes, y morbilidad y mortalidad. Además, Pargament y Mahoney (2002), refieren que la American Psychological Association ha definido la religiosidad como una variable de la diversidad cultural.

Todo esto reafirma la idea de que no puede descuidarse la dimensión espiritualreligiosa al evaluar, diagnosticar y tratar a las personas. No menos importante que la investigación de las implicancias prácticas es el estudio teórico acerca de la relación, semejanzas y diferencias entre espiritualidad y religión, y éste aún no ha terminado. Como Peterson y Seligman (2004) plantean, al momento presente, en este campo se continúa pensando la espiritualidad y la religiosidad como conceptualmente distintas pero funcionalmente idénticas. Y falta por determinar si estas experiencias se asocian a distintos resultados, es decir, si efectivamente son también diferentes funcionalmente.

\section{Influencia de la espiritualidad en la salud y el bienestar}

Delgado (2005) plantea que una fuerte conexión espiritual puede mejorar la satisfacción con la vida o facilitar el adaptarse a los impedimentos. Pargament (1997, citado en Clark, 2004), encontró que las creencias religiosas y espirituales contribuyen a la habilidad para enfrentar efectivamente con la enfermedad, discapacidad y eventos vitales negativos.

Otros hallazgos asociados a la influencia de la espiritualidad en la salud física, señalan que las personas que regularmente asisten a la iglesia, oran individualmente y leen la Biblia, tienen una presión sanguínea diastólica mucho más baja que los menos religiosos, sufren menos hospitalizaciones, tienden a tener estilos de vida más saludables, tienden a evitar el abuso de alcohol y drogas y los comportamientos sexuales riesgosos. Las personas que asisten regularmente a servicios religiosos tienen sistemas inmunes más fuertes que sus contrapartes menos religiosos, y muestran significativamente mejores resultados al sufrir alguna enfermedad que los no religiosos (Koenig, 2001).

Con relación al efecto positivo en la salud física y mental que las dimensiones religiosas y/o espirituales tienen en el tratamiento médico, se ha encontrado que, en pacientes oncológicos, la espiritualidad mejora la función inmune, aumenta la sobrevida, disminuye los síntomas de la enfermedad y los efectos adversos del tratamiento, favorece estilos de vida y comportamientos más sanos, y por lo tanto contribuye a un menor riesgo de enfermedades, y en general, mejora la calidad de vida. Por estas razones, Navas, Villegas, Hurtado, y Zapata (2006) concluyen que se justifica la inclusión de la espiritualidad en los programas de apoyo psicosocial dirigidos a pacientes oncológicos.

Un estudio de religiosidad y bienestar en pacientes de una clínica geriátrica encontró que aquellos que no eran activos religiosamente tenían mucho más altos niveles de uso de alcohol y tabaco, depresión, ansiedad y cáncer, que aquellos que eran muy activos 
religiosamente, los que además, disfrutaban de buena salud física y mental (Karren et al., 2002, citados en Clark, 2004).

A partir de sus estudios, Koenig (2001) señala que la fe religiosa parece proteger a los adultos mayores de las dos más importantes aflicciones del final de la vida, la enfermedad cardiovascular y el cáncer, y también parece evitar por mayor tiempo el llegar a una invalidez.

Rivera-Ledesma y Montero-López (2005) citan distintos estudios que encuentran efectos de alivio del estrés de la hospitalización, la enfermedad, y las pérdidas típicas de la vejez, asociación con una menor mortalidad en casos crónicos, predicción positiva de salud mental y de satisfacción vital, y una asociación negativa entre espiritualidad y depresión.

Un estudio que investigó los efectos de la participación en el servicio religioso verificó, en personas evaluadas en dos momentos con un lapso de entre dos y tres años, que dentro de los beneficios de una más frecuente participación religiosa, se encontraba una menor proporción de depresión, y otro estudio, sobre el bienestar en adultos de tres generaciones, encontró que a mayor frecuencia en la concurrencia al servicio religioso hubo menores índices de depresión, al ser evaluados once años después. Similares resultados se hallaron en satisfacción con la vida y felicidad, evaluadas trece años más tarde (Levin, 2001).

Otros beneficios que aporta la espiritualidad tienen que ver con la longevidad, el enfrentamiento a la muerte y la satisfacción vital. Koenig (2001) encuentra que los adultos mayores que tienen una profunda y personal fe religiosa tienen una mayor sensación de bienestar y satisfacción vital que sus pares menos religiosos. Asimismo, encuentra que los mayores que tienden a confiarse más a su fe religiosa y a la oración cuando se encuentran bajo estrés, tienden a mostrar mucho menor o ningún temor a la muerte, comparados con pares para quienes la fe y la oración son menos importantes.

Levin (2001), aporta evidencia de que la participación en actividades religiosas públicas, a través de membrecías religiosas, beneficia la salud ya que se promueven conductas y estilos de vida más saludables, y también, porque brindan un apoyo que amortigua los efectos del estrés y el aislamiento. Del mismo modo, los adultos mayores más involucradas en sus comunidades religiosas, también reportaron menor temor a la muerte. No obstante, más que la actividad religiosa en sí, señala Koenig (2001), es la fe personal la que parece ayudar a las personas mayores a enfrentar el estrés y el temor a la muerte. Por último, las personas mayores son más sanas físicamente en la última parte de sus vidas y viven más que sus contrapartes menos religiosos (Koenig, 2001).

La espiritualidad y religiosidad no sólo son fuente de apoyo y beneficios, también pueden ser fuente de conflictos y sufrimiento. Kliewer y Staultz (2006), explican cómo la vida espiritual puede dejar de ser saludable. Estos autores plantean que no es infrecuente que en personas profundamente espirituales surjan fuertes sentimientos de culpa, desesperanza, ira, y falta de sentido, que son la contraparte dolorosa de la espiritualidad, y que ha sido descrita como ‘el desierto' y ‘la noche oscura del alma'. 
En estos momentos o etapas, las personas religiosas necesitan especialmente de un apoyo informado que brinde una real comprensión.

\section{Envejecimiento positivo y espiritualidad}

Los autores hablan de envejecimiento positivo como opuesto a envejecimiento patológico y distinto del envejecimiento normal. Muñoz (2002) explica que el envejecimiento normal, o senescencia, alude a la etapa del ciclo vital en la cual se aprecian cambios biológicos, psicológicos y sociales, y decrementos benignos propios de la edad. Y a pesar de que el riesgo de padecer enfermedades aumenta con la edad, rechaza la noción antigua de un deterioro universal e irreversible. La noción de envejecimiento patológico, también denominado senilidad, corresponde a la concepción biomédica tradicional del desarrollo humano, que enfatiza las disfunciones observadas en el ser que envejece, y plantea que con la edad aparecen en el individuo enfermedades crónicas.

Una visión más positiva y reciente del envejecimiento humano plantea que, paralelo al deterioro benigno del adulto mayor se da la evolución y desarrollo de otros aspectos que se mantienen a pesar de los decrementos, visión que ha llevado a hablar de envejecimiento exitoso, competente, positivo, saludable, funcional, satisfactoria, buen envejecer, etc.

Para Villar, Triadó, Resano, y Osuna (2003), un envejecimiento exitoso ocurriría cuando las personas sienten satisfacción por poder adaptarse a las situaciones cambiantes de su vida. Un estudio de Ryff (1987, citada por Schaie y Willis, 2003) encuentra que el bienestar es definido por los adultos mayores como algo relacionado con ser una persona que cuida a los demás, que es compasiva y que tiene buenas relaciones con otros y, por su parte, como criterios para un buen envejecimiento, los adultos mayores enfatizan la aceptación del cambio, el sentido del humor y el disfrutar de la vida

Para Schaie y Willis (2003), un envejecimiento óptimo implica una estrategia general de selectividad con los esfuerzos que se realizan y usar estrategias y actividades alternativas para compensar las pérdidas que conlleva el proceso de envejecimiento. En este sentido, se ha planteado que las personas no suelen usar todo su potencial y que ello no se aprecia en la juventud, pero en la vejez destacan los individuos que funcionan cerca de sus límites y maximizan las cualidades de apoyo de su entorno, con lo que envejecen mucho mejor y parecen ser más competentes que otros (Schaie y Willis, 2003).

Vaillant (2002) señala que Erikson parece ser el primer autor que se ocupa del envejecimiento y la vejez desde un punto de vista psicológico, conceptualiza el desarrollo en la adultez como progreso y no como declinación, e incorpora la dimensión espiritual en su enfoque de la última parte de la vida. Para Erikson (1966), en la tercera edad, ocurriría un conflicto entre integridad y desesperanza. Se lograría la integridad en la medida que al envejecer, la persona logra adaptarse a los triunfos y desilusiones que implica haber dado vida a otros seres humanos, y haber producido cosas e ideas, y se caracteriza por un sentimiento de seguridad y satisfacción acumulada, de haber 
alcanzado un orden en el mundo y un sentido espiritual, que es la aceptación del propio ciclo de vida como algo que debía ser de esa manera (Erikson, 1966).

Una resolución favorable del conflicto daría como resultado las fortalezas de sabiduría y renunciamiento, entendidas como una posición informada y desapegada por la vida misma, frente a la muerte misma. En otras palabras, una capacidad de captar y comprender, con la perspectiva de la experiencia, el mundo circundante y sus circunstancias, posibilidades y limitaciones (Erikson, 2000). La falta o pérdida de esta integración, cuando el individuo no acepta el propio ciclo vital como lo esencial de la vida, se expresa en temor a la muerte y desesperanza.

Erikson (1966) no habla de envejecimiento positivo propiamente tal, pero plantea que un individuo maduro debe haber desarrollado en grado suficiente todas las fortalezas psicosociales que resultan del desarrollo desde el nacimiento, y que son impulso y esperanza, autocontrol y voluntad, dirección y propósito, método y capacidad, devoción y fidelidad, afiliación y amor, producción y cuidado, y renunciamiento y sabiduría.

30 años después de publicada su teoría, Erikson (2000) añade una última etapa, pues señala que la vejez a los 80 y 90 años conlleva nuevas exigencias, revalorizaciones y dificultades diarias que sólo podrían comprenderse adecuadamente contemplando una novena etapa. En ésta, cobran gran importancia las fortalezas desarrolladas previamente, las características de personalidad y aprendizajes hacia la madurez, ya que permiten afrontar más positivamente los aspectos difíciles de esta novena etapa, en la que ocurriría una amenaza sobre los logros de toda una vida, sobre la autonomía, la iniciativa, etc., en su mayoría, provocados por la debilitación física progresiva. Los logros de esta última etapa serían la esperanza (al igual que en la primera) y la fe.

En términos de predecir un envejecimiento exitoso, los resultados del Study of Adult Development, de la Universidad de Harvard, el más prolongado estudio del envejecimiento en el mundo, han permitido identificar siete factores. Estos son tener un matrimonio afectuoso, no abusar del alcohol, no fumar mucho, hacer ejercicio físico, mantener un peso adecuado, y poseer buenos mecanismos de afrontamiento. Así, la satisfacción con la propia vejez parece depender de las opciones que haya escogido cada persona, sumadas a buenos mecanismos de afrontamiento. Otros resultados de esta investigación sugieren que, más que el dinero o el status social, lo que marca la diferencia para un envejecimiento exitoso es la educación y una participación activa en una familia numerosa (Vaillant \& Mukamal, 2001).

En estos estudios, Vaillant (2002) ha encontrado que la espiritualidad y la religiosidad no aumentan en la vejez. Este autor señala que las personas tienden a mantener en la vejez las creencias y hábitos de toda su vida. Pero aclara que, así como envejecer no conduce inevitablemente al desarrollo espiritual, sí altera las condiciones de la vida en formas que conducen a la espiritualidad. El envejecer disminuye los ritmos y deja tiempo y espacio para disfrutar de cosas simples. Simplifica la rutina diaria. Facilita la aceptación de las cosas que no se pueden cambiar. Envejecer disminuye las pasiones y aumenta la capacidad de estar calmado interiormente. Lleva a contemplar la muerte y 
a familiarizarse con el dejar de ser. Encamina hacia llegar a ser uno con la naturaleza última de todos los seres vivos (Vaillant, 2002).

Vaillant (2002) encontró también que las personas más profundamente comprometidas con creencias religiosas o espirituales no tienen envejecimientos más exitosos, y en cambio, tienen cuatro veces más posibilidades que los no religiosos o espirituales, de haber experimentado una depresión, y el doble de veces de tener varios parientes con depresión. En el mismo sentido, encontró una positiva y significativa relación entre la fuerza de la afiliación religiosa y una historia de depresión en el pasado, y ésta, a su vez, correlacionaba negativamente con la riqueza del apoyo social disponible y con ser saludable y feliz (Vaillant, 2002).

Además de un envejecimiento exitoso, Vaillant (2002) plantea que un envejecimiento positivo tiene que ver con gratitud, perdón y alegría, y más aún, un envejecer que denomina 'con gracia', tiene que ver con seis grandes tareas o quehaceres: 1) cuidar a otros, estar abierto a nuevas ideas, y mantenerse útil socialmente, 2) mantener la integridad postulada por Erikson (1966): aceptar el pasado y pasar momentos en él para nutrirse de los logros pasados, junto con seguir dispuesto a aprender de la generación siguiente, 3) mantener las demás fortalezas de la teoría de Erikson (1966): esperanza en la vida, esforzarse por ser todo lo autónomo posible, y valorar toda iniciativa, 4) disfrutar de la vida, mantener el sentido del humor y la capacidad para jugar, 5) ser tolerante con los aspectos desagradables de la vejez, cuidarse, aceptar las necesidades de dependencia y agradecer el apoyo recibido, y 6) tratar de mantener contacto e intimidad con viejos amigos y lograr hacer nuevas amistades.

Aunque Vaillant (2002) no ofrece una definición de espiritualidad, muchos de los conceptos que asocia al envejecimiento positivo o con gracia, como la gratitud, la tolerancia, la esperanza, la bondad y generosidad, son aspectos que otros autores consideran como factores o elementos característicos de la espiritualidad.

En contraste con los resultados de Vaillant (2002), otros estudios encuentran que la espiritualidad ocupa un lugar central en un buen proceso de envejecer, y se han documentado beneficios importantes de la vida espiritual en la salud y bienestar del adulto mayor, tal como se ha expuesto más arriba.

Tornstam (2005) critica el frecuente desencuentro entre la teoría y los datos empíricos, llamando la atención sobre el hecho que cuando estos últimos no apoyan la teoría, se critica al estudio para mantener intacta la teoría. Para este autor, este es uno de los factores que han llevado a proyectar en la vejez, los valores, patrones de actividad y expectativas de la mediana edad, y luego, definir estos valores, patrones y expectativas como un envejecimiento exitoso. Y agrega que estas proyecciones, además de estar enraizadas en la edad mediana, también lo están en la cultura occidental y en la esperanzas que los occidentales blancos exitosos de clase media tienen para que el éxito continúe en la vejez. Estos valores suelen enfatizar la actividad, productividad, individualismo, independencia, riqueza, salud, sociabilidad, etc.

Tornstam (2005) sugiere que el envejecer tiene su propio significado y carácter, y que hay un desarrollo continuo en la vejez, por lo que no concuerda con las ideas de la 
vejez caracterizada por estabilidad y continuidad, en lugar de desarrollo y cambio. Para descubrir estos aspectos descuidados u ocultos de la vejez, el autor se propone escuchar lo que las personas mayores tienen que decir. Así, utilizando tanto metodologías cualitativas como cuantitativas, llega a los resultados que conforman su teoría.

La teoría que formula Tornstam (2003) plantea que hay un proceso propio del envejecimiento, que tienden a desarrollar los individuos en la última etapa de sus vidas, y que se caracteriza por un potencial general hacia la gerotrascendencia. Esta consiste en un cambio en la metaperspectiva, desde una visión de mundo materialista y pragmática a una más cósmica y trascendente, acompañada habitualmente de un incremento en la satisfacción vital. La gerotrascendencia sería el estadio final de un proceso natural hacia la madurez y la sabiduría, que no necesariamente está asociada a creencias o prácticas religiosas.

De acuerdo con esta teoría, el individuo gerotrascendente experimenta una nueva comprensión acerca de cuestiones existenciales fundamentales; a menudo un sentimiento de comunión cósmica con el espíritu del universo, una redefinición del tiempo, del espacio, de la vida y la muerte, así como una redefinición de sí mismo y de su relación con otros. El individuo se vuelve menos preocupado por sí mismo y más selectivo en sus elecciones sociales o de otra clase. Ocurre un sentimiento aumentado de afinidad con las generaciones pasadas, un menor interés en interacciones sociales superfluas, y se vuelven más importantes los momentos de aislamiento positivo (Tornstam, 2003).

Las dimensiones de la gerotrascendencia fueron descritas como cambios ontológicos acerca del cosmos, de sí mismo, y de las relaciones sociales e individuales, y operacionalizadas respectivamente en tres medidas: trascendencia cósmica, coherencia y necesidad de soledad. En tres distintos estudios realizados por Tornstam (2003), utilizando métodos cuantitativos y cualitativos, uno con individuos daneses y dos con individuos suecos, entre 1994 y 2001, con un total de unos 3.600 sujetos, se encontró que todas las dimensiones de la gerotrascendencia aumentan con la edad. La trascendencia cósmica y la coherencia comenzarían durante la primera mitad de la vida adulta y se desarrollan gradualmente hasta su máximo hacia el final de la vida. La necesidad de soledad aparece en su punto máximo hacia el final de la vida, pero se desarrolla más rápidamente durante la primera mitad de la vida adulta (Tornstam, 2003).

A diferencia del modelo de desarrollo de Erikson (1966; 2000), que termina con un alto grado de madurez o integridad del yo, entendida como una integración y aceptación de los distintos aspectos de toda la vida, en retrospectiva, el modelo de gerotrascendencia de Tornstam (2005), plantea una dirección más hacia adelante o hacia afuera, que incluye una redefinición de la realidad.

Este autor aclara que la gerotrascendencia es más bien un semilla que puede, en las condiciones adecuadas, germinar y desarrollarse, y no una pauta que se cumpla en cada caso. En este sentido, no rechaza la importancia de la integridad del ego en la teoría de Erikson, ni la importancia de la actividad para la satisfacción vital, sino que agrega un modelo que no había sido considerado (Tornstam, 2005). 
Un estudio que buscó conocer la percepción de un grupo de individuos de la tercera edad acerca de la teoría de la gerotrascendencia, utilizando métodos cualitativos, encontró que la experiencia de envejecer de todos los participantes correspondía de alguna manera a la descripción teórica y que, en su percepción, esta descripción del envejecer estaba más o menos de acuerdo con su propio envejecimiento. Consideraron también beneficioso discutir acerca de la teoría, pues les brindaba una visión más positiva del envejecimiento que les estimulaba a seguir siendo tal cual eran (Wadensten, 2005).

\section{Espiritualidad y práctica clínica con adultos mayores}

Dos aspectos relevantes en lo que respecta al ejercicio clínico con adultos mayores son los prejuicios negativos hacia la vejez, y la consideración de las creencias espirituales de los pacientes mayores.

En primer lugar, respecto del prejuicio negativo hacia la vejez en la persona del profesional que atiende, así como cada sociedad produce un tipo de anciano, le define un papel y una imagen, nuestra cultura occidental ha tendido a relevar los aspectos negativos de la vejez, y la ha pensado triste, achacosa y enferma (Álvarez, 1998).

Consecuencia de esta realidad socialmente construida es que, desde la infancia, las personas asumen estos valores y actitudes negativos de la sociedad hacia la vejez, y luego cuando viejos, tienden a adaptarse a la imagen que han construido. Y aunque esto no impide, sí limita en gran medida las posibilidades de ser viejo de otra manera y de concebir la vejez de otra manera. Por esta razón, debe pensarse al profesional que trabaja con adultos mayores, criado en la misma sociedad, y expuesto por tanto a los mismos prejuicios, que influyen no sólo en la forma en que encare su propia vejez sino también, naturalmente, a sus pacientes..

El problema puede estar en que bajo los conocimientos y buenas intenciones, los prejuicios hayan quedado ocultos. Una reflexión atenta de los propios ocultos prejuicios puede resultar útil, dadas las muy diversas formas que pueden tomar: idealización, negación, sobreprotección, etc. Afirmaciones tales como “¿Viejo, Ud?, ipara nada!. ¡Todo lo contrario, se ve Ud. cada día más joven!”, “¡Si se es joven de espíritu, entonces estamos bien, porque el alma no envejece!”, aunque a una primera impresión pueden sonar como alegres o positivas expresiones hacia un paciente anciano, revelan en el fondo la imagen de la vejez como algo malo, feo, que hay que evitar o esconder.

Así como cualquier etapa de la vida, la vejez también presenta conflictos y sufrimiento, pero a ellos se suma el enfrentar la propia muerte y la evaluación de la vida que suele ocurrir hacia el final de la misma (Erikson, 1966). Koenig (2001) cita estudios geriátricos y psiquiátricos que han mostrado que el temor a la muerte puede causar un estrés crónico que puede llevar a una significativa disminución de la calidad de vida de los adultos mayores. La ayuda que ofrece el cínico se fortalece entonces, si puede intervenir en los temores y angustias del paciente, también desde sus propias concepciones espirituales o religiosas que éste tiene acerca de lo que hay más allá de la muerte, o de sus propias respuestas al por qué y para qué de la vida. 
Entonces, puesto que aunque puede parecer natural que la espiritualidad sea considerada en la intervención psicológica del mismo modo que cualquier otro tipo de creencia o valor, ello no ocurre así. Como señalan Rivera-Ledesma y Montero-López (2007), los psicólogos están recién comenzando a reconocer la importancia del fenómeno espiritualidad en la naturaleza humana. La inclusión de lo espiritual en el tratamiento psicológico es un esfuerzo reciente cuya consolidación no se ha logrado aún, y la psicología académica parece aceptar lo espiritual aún más lentamente que la clínica.

El papel que asumen ante las necesidades del paciente las distintas disciplinas de la medicina, enfermería, y salud mental, se han definido de modo que, aunque algunas tareas pueden traslaparse, otras funciones, dado su alto grado de especialización, permanecen bajo el dominio de su respectiva y específica profesión. No obstante, ninguna profesión puede apropiarse del campo de lo espiritual, ni siquiera los representantes de lo sacro, pues es el paciente quien demanda un espacio de intimidad espiritual a quien cree que puede dárselo, sin importar su profesión (Rivera-Ledesma \& Montero-López, 2007), y también porque la espiritualidad es tan parte de la personalidad, como de la sociedad, como de la familia.

Siguiendo a Rivera-Ledesma y Montero-López (2007), la cuestión es que cada uno, médico, enfermero, psicólogo, psiquiatra, o líder espiritual, deben estar preparados para saber responder a ese llamado. Y aquí, el problema del ejercicio clínico en lo espiritual es doble. Por una parte, la formación del clínico, que va más allá de la formación profesional pues pasa por su personalidad y sus actitudes de fondo hacia lo espiritual. Y por otra, sus habilidades profesionales, pues no sólo se necesita ser sensible a lo espiritual, también se debe estar capacitado para intervenir espiritualmente o dirigir espiritualmente a un paciente en el contexto de sus creencias y prácticas religioso-espirituales.

Resulta comprensible entonces, que al profesional de la salud le cueste trabajo enfrentar lo espiritual con sus pacientes, pues, en primer lugar, no suele haber sido formado para ello, además de que no necesariamente comparte las creencias de sus pacientes, y aún en el caso de que las compartiera, es complejo el límite ético.

Zinnbauer y Pargament (2000, citados en Rivera-Ledesma \& Montero-López, 2007) sugieren que existen cuatro aproximaciones a lo religioso y espiritual en la literatura del ámbito clínico, aplicadas al papel de la religión en la consejería, y las denominan rechacista, exclusivista, constructivista y pluralista. La aproximación rechacista niega de plano las realidades sagradas fundamentales de las creencias de sus adherentes, tales como la existencia de Dios. Esta postura reduce la religión a una defensa o alteración psicológica.

La aproximación exclusivista, está basada en la dimensión religiosa o espiritual de la existencia, y toma la palabra de Dios en las escrituras como la única verdadera concepción de la naturaleza y la conducta humana. Esta postura sólo sirve para una población con valores similares, pues si el cliente no tiene esta visión, sería obligación del exclusivista llevarlo a ella. 
La aproximación constructivista niega la existencia de una realidad absoluta, pero reconoce la habilidad de los individuos para construir sus propias realidades y significados. Aquí el trabajo es conducido dentro del sistema de creencias del cliente. Advierten los autores que como es posible que las creencias personales del constructivista difieran completamente de las del cliente, puede ocurrir que la manipulación de las metáforas y símbolos sagrados del cliente para un fin secular, sean un acto insincero, en un contexto en que se debe ser exactamente lo contrario.

La aproximación pluralista reconoce la existencia de una realidad absoluta espiritual y permite múltiples interpretaciones y formas de acceder a ella. Reconoce que esta realidad se expresa en diferentes culturas, por diferentes personas y de diferentes maneras, y puede usar múltiples índices de patología y salud. El clínico y el cliente deben estar conscientes de sus historias religiosas o espirituales, marcos conceptuales y valores, para trabajar juntos en la definición las metas de la terapia.

Para Zinnbauer y Pargament (2000, citados en Rivera-Ledesma \& MonteroLópez, 2007), las aproximaciones constructivista y pluralista permitirían tratar un amplio rango de aspectos religiosos y espirituales de una manera efectiva y respetuosa.

Con base en los planteamientos anteriores, es posible sostener por una parte, que la consideración de los aspectos espirituales de los adultos mayores en la atención clínica resulta de gran relevancia para una correcta comprensión de sus angustias, problemas o sufrimiento, dadas las particulares características que adquieren estas en los procesos de enfrentamiento a la muerte y de evaluación de sus vidas. Por otra parte, y para intervenir adecuadamente con adultos mayores, incluyendo el trabajar con su espiritualidad, un requisito indispensable debe ser una adecuada formación profesional y personal, que incorpore capacitación en la diversidad religiosa y espiritual, a fin de asegurar competencia en esa área.

\section{Conclusiones y Comentarios}

Ante una mayor expectativa de vida y una sociedad que envejece, muchas características asociadas al adulto mayor están requiriendo redefinición, en particular el concepto, las expectativas, la imagen y el rol de los adultos mayores. Es posible que en poco tiempo lo que hoy se sabe o cree saber acerca de la tercera edad ya no corresponda a la realidad. Y como las teorías se mantienen vigentes en la medida que resultan útiles como herramientas para explicar, predecir y controlar, se debe estar realmente dispuestos a probar si las que tenemos siguen sirviendo o deben ser complementadas, mejoradas o definitivamente reemplazadas.

El proceso de envejecer no es necesariamente un apacible retiro de la vida a disfrutar de los logros alcanzados en espera de una pacífica muerte, ni tampoco una lucha por mantenerse vigente en un mundo de jóvenes. Pero puede serlo. El envejecimiento no está preestablecido. Es una etapa y proceso de desarrollo más en la vida. Y a pesar de los hitos normativos más o menos comunes, como la jubilación, la llegada de nietos, o 
bisnietos, cierta disminución de las redes sociales, la muerte de amigos o de la pareja, etc., hay tantas formas de envejecer como estilos de vida.

Como en cualquier otra etapa del desarrollo, en la vejez hay alegrías y penas, conflictos y soluciones, conquistas y derrotas. Hasta ahora los psicólogos hemos entrado mayormente en el terreno de los conflictos, las penas y las derrotas, buscando naturalmente aliviar el sufrimiento. Pero la psicología positiva está haciendo un llamado a considerar también los aspectos positivos, sanadores y enriquecedores, junto con, no en lugar de, los aspectos más negativos. En este ánimo de equilibrar la mirada, es que se ha querido abordar el tema de la espiritualidad y del envejecimiento en este trabajo. Considerando que la espiritualidad es una dimensión humana como cualquier otra, necesaria de considerar si es que está presente en la vida de la persona que demanda ayuda, y que el envejecimiento es una parte de la vida que vale la pena vivir bien.

Muchas características se han encontrados asociadas a envejecer bien, óptimo y con gracia. Gratitud, perdón y alegría. Cuidar a otros, integridad, esperanza en la vida, disfrutar de la vida, sentido del humor, tolerancia, cuidarse, aceptar las propias limitaciones, contacto e intimidad (Vaillant, 2002). Las mismas características ayudarían a vivir bien casi cualquier etapa de la vida. Pero no necesariamente pueden desarrollarse en cualquier otra etapa de la vida. La madurez que permite el paso del tiempo aun cuando no las desarrolla por sí sola, sí contribuye o permite hacerlo.

Los hallazgos acerca de los beneficios de la espiritualidad en la tercera edad son contradictorios. Vaillant (2002) encuentra que en la vejez ni se produce un aumento de la espiritualidad, ni es favorecida la forma de envejecer con la espiritualidad. Para este autor, son las relaciones interpersonales, los vínculos, informales, familiares y sociales, los que favorecen un envejecimiento exitoso. Sin embargo, otros autores han encontrado beneficios de la espiritualidad en la salud, alivio del estrés de la hospitalización, salud mental, bienestar, enfrentamiento a la muerte, etc. (Delgado, 2005; Koenig, 2001; Levin, 2001; Navas, Villegas, Hurtado, \& Zapata, 2006; RiveraLedesma \& Montero-López, 2005).

Un autor en particular desarrolla una teoría (Tornstam, 2003; 2005) que considera una forma distinta de envejecer y que no había sido considerada. En esta teoría, de la gerotrascendencia, un aspecto central del envejecimiento es la espiritualidad, pues encuentra en los adultos mayores una nueva comprensión acerca de cuestiones existenciales fundamentales; un sentimiento de comunión cósmica con el espíritu del universo, una redefinición del tiempo, del espacio, de la vida y la muerte, y una redefinición de sí mismo y su relación con otros.

Entonces, no hay consenso, hay diversidad. Para un buen envejecimiento puede ser central el cuidado de los vínculos interpersonales significativos que sugiere Vaillant (2002) (tanto si sus hallazgos son equivalentes a lo que para otros autores se denomina espiritualidad como si no), y también para un buen envejecimiento puede ser central el proceso de trascendencia de lo terreno, que puede suponer una fe en Dios, o una comunión cósmica con un universo contenedor. Pueden haber además otras maneras de envejecimiento positivo por conocer. En cualquier caso, el profesional que atiende 
a la persona que envejece, debe poder captar lo que para cada caso es central, para planificar y dirigir desde allí la mejor indicación terapéutica.

Todas las teorías confluyen en la común aspiración a lograr el mejor envejecimiento posible, dentro de los valores y posibilidades que se conocen. En este trabajo se ha querido fundamentar la pertinencia de incorporar la dimensión de la espiritualidad en el buen envejecimiento, y en el buen asesoramiento o tratamiento de los adultos mayores que tienen creencias religiosas o espirituales. La psicología positiva nos recuerda que puede ser provechoso considerar, no sólo los indicadores de patología, sino también los indicadores de salud, de modo de lograr una mirada más equilibrada o más completa, que sepa reconocer tanto las riquezas como los padecimientos del envejecimiento.

\section{Referencias}

Álvarez, M. (1998). El concepto de vejez. En G.A. Ferrero (comp.). Envejecimiento y vejez. Buenos Aires: Atuel.

Clark, R. (2004). Religiousness, Spirituality, and IQ: Are They Linked? Explorations: An Undergraduate Research Journal. Extraído el 12 de abril del 2007 de: http:// undergraduatestudies.ucdavis.edu/explorations/2004/clark.pdf

Delgado, C. (2005). A Discussion of the Concept of Spirituality. Nursing Science Quarterly, 18(2), 157-162

Erikson, E. (1966). Infancia y Sociedad. $2^{\text {a }}$ ed. Buenos Aires: Hormé.

Erikson, E. (2000). El ciclo vital completado. Ed. revisada y ampliada. Barcelona: Paidós.

Green, R., Benshoff, J., \& Harris-Forbes, J. (2001). Spirituality in Rehabilitation Counselor Education: A Pilot Survey. Journal of Rehabilitation, July-Sept.

Kliewer, S. \& Saultz, J. (2006). Health care and spirituality. United Kingdom: Radcliffe.

Koenig, H. (2001). The healing power of faith. New York: Touchstone.

Levin, J. (2001). God, faith and health. Exploring the spirituality-healing connection. New York: John Willey \& Sons.

Muñoz, J. (2002). Psicología del envejecimiento. Madrid: Pirámide.

National Cancer Institute (s/f). Dictionary of Cancer Terms. Recuperado el 2 de Julio de 2007 de: http://www.cancer.gov/Templates/db_alpha.aspx?CdrID=441265

Navas, C., Villegas, H., Hurtado, R. \& Zapata, D. (2006). La conexión mente-cuerpoespíritu y su efecto en la promoción de la salud en pacientes oncológicos. Revista Venezolana de Oncología, 18(1), 28-37.

Pargament, K. \& Mahoney, A. (2002). Spirituality. En C. R. Snyder \& Sh. Lopez. Handbook of Positive Psychology. New York: Oxford University Press. 
Peterson, Ch. \& Seligman, M. E. P. (2004). Character strenghts and virtues. A handbook and classification. Washington D. C.: American Psychological Association.

Rivera-Ledesma, A. \& Montero-López, M. (2005). Espiritualidad y religiosidad en adultos mayores. Salud Mental (28)6.

Rivera-Ledesma, A. \& Montero-López, M. (2007). Ejercicio clínico y espiritualidad. Anales de Psicología, 23(1), 125-136.

Schaie, K. W. \& Willis, S. (2003). Psicología de la edad adulta y la vejez. $5^{\mathrm{a}}$ ed. Madrid: Pearson Prentice Hall.

Spilka, B. (2003). The psychology of religion: An empirical approach. New York: Guilford.

Tonon, G. (comp.). (2006). Juventud y protagonismo ciudadano. Buenos Aires: Espacio. Tornstam, L. (2003). Gerotranscendence from young old age to old old age, Online publication from The Social Gerontology Group, Uppsala.

Tornstam, L. (2005). Gerotrascendence. A developmental theory of positive aging. New York: Springer.

Vaillant, G. (2002). Aging well. New York: Little, Brown \& Company.

Vaillant, G. \& Mukamal, K. (2001). Succesfull aging. American Journal of Psychiatry, (158), 839-847.

Villar, F., Triadó, C., Resano, C. S., \& Osuna, M. J. (2003). Bienestar, adaptación y envejecimiento: cuando la estabilidad significa cambio. Revista Multidisciplinaria de Gerontología, (13)3, 152-162.

Wadensten, B. (2005). Introducing older people to the theory of gerotranscendence. Journal of Advanced Nursing, 52 (4), 381-388. 
Psicodebate 8. Psicología, Cultura y Sociedad - Noviembre 2007 